\title{
RETRACTION
}

doi:10.1038/nature06819

\section{Genetic tracing reveals a stereotyped sensory map in the olfactory cortex}

Zhihua Zou, Lisa F. Horowitz, Jean-Pierre Montmayeur, Scott Snapper \& Linda B. Buck

Nature 414, 173-179 (2001)

This Article described patterns of labelling observed in olfactory cortex when a transneuronal tracer was co-expressed with single odorant receptor genes in the mouse olfactory epithelium. During efforts to replicate and extend this work, we have been unable to reproduce the reported findings. Moreover, we have found inconsistencies between some of the figures and data published in the paper and the original data. We have therefore lost confidence in the reported conclusions. We regret any adverse consequences that may have resulted from the paper's publication.

Author Contributions L.B.B. and L.F.H. conceived the project, L.F.H. and J.-P.M. prepared gene-targeting constructs to generate the mice, S.S. trained Z.Z. in gene-targeting techniques, Z.Z. prepared and analysed the mice and provided all figures and data for the paper, and L.B.B. and Z.Z. wrote the paper. Correspondence and requests for materials should be addressed to L.B.B. (lbuck@fhcrc.org).

\section{CORRIGENDUM}

\section{doi:10.1038/nature06818}

\section{Behavioural improvements with thalamic stimulation after severe traumatic brain injury}

N. D. Schiff, J. T. Giacino, K. Kalmar, J. D. Victor, K. Baker, M. Gerber, B. Fritz, B. Eisenberg, T. Biondi ${ }^{1}$, J. O'Connor, E. J. Kobylarz, S. Farris, A. Machado, C. McCagg, F. Plum, J. J. Fins \& A. R. Rezai

'JFK Johnson Rehabilitation Institute, Edison, New Jersey 08818, USA.

Nature 448, 600-603 (2007)

In this Letter, Tracey Biondi was omitted from the author list. In addition, a sentence in the Author Contributions statement should be revised to read: 'M.G., B.F., B.E., T.B. and J.O. collected behavioural data and assisted in the development of secondary outcome measures.'. 